\title{
Three-dimensional microscopic analysis of clinical prostate specimens
}

\author{
Martin E van Royen, ${ }^{1,2}$ Esther I Verhoef, ${ }^{1}$ Charlotte F Kweldam, ${ }^{1}$ Wiggert A van Cappellen, ${ }^{1,2}$ \\ Gert-Jan Kremers, ${ }^{1,2}$ Adriaan B Houtsmuller ${ }^{1,2}$ \& Geert J L H van Leenders ${ }^{1}$ \\ ${ }^{1}$ Department of Pathology, and ${ }^{2}$ Erasmus Optical Imaging Centre, Erasmus Medical Centre, Rotterdam, the Netherlands
}

Date of submission 21 April 2016

Accepted for publication 23 June 2016

Published online Article Accepted 29 June 2016

van Royen M E, Verhoef E I, Kweldam C F, van Cappellen W A, Kremers G-J, Houtsmuller A B \&

van Leenders G J L H

(2016) Histopathology. DOI: 10.1111/his.13022

Three-dimensional microscopic analysis of clinical prostate specimens

Aims: Microscopic evaluation of prostate specimens for both clinical and research purposes is generally performed on 5 - $\mu$ m-thick tissue sections. Because cross-sections give a two-dimensional (2D) representation, little is known about the actual underlying three-dimensional (3D) architectural features of benign prostate tissue and prostate cancer $(\mathrm{PCa})$. The aim of this study was to show that a combination of tissue-clearing protocols and confocal microscopy can successfully be applied to investigate the 3D architecture of human prostate tissue.

Methods and results: Optical clearing of intact fresh and formalin-fixed paraffin-embedded (FFPE) clinical prostate specimens allowed us to visualize tissue structures up to a depth of $800 \mu \mathrm{m}$, whereas, in uncleared tissue, detection of fluorescence was only possible up to $70 \mu \mathrm{m}$. Fluorescent labelling with a general nuclear dye and antibodies against cytokeratin (CK) 5 and CK8-18 resulted in comprehensive 3D imaging of benign peripheral and transition prostate zones, as well as individual PCa growth patterns. After staining, clearing, and imaging, samples could still be processed for 2D (immuno)histochemical staining and DNA analysis, enabling additional molecular and diagnostic characterization of small tissue specimens.

Conclusions: In conclusion, the applicability of 3D imaging to archival FFPE and fresh clinical specimens offers unlimited opportunities to study clinical and biological topics of interest in their actual 3D context.

Keywords: fluorescence microscopy, pathology, prostate, three-dimensional (3D), tissue clearing

\section{Introduction}

The Gleason grade is an important parameter for therapeutic decision-making and predicting the outcome of patients with clinical prostate cancer (PCa), and is entirely based on classification of architectural tumour growth patterns. ${ }^{1}$ Pathological investigation for both diagnostic and research purposes is routinely carried out by microscopic evaluation of $4-5-\mu \mathrm{m}$ -

Address for correspondence: Dr M E van Royen, Department of Pathology/Erasmus Optical Imaging Centre (OIC), P.O. Box 2040, 3000 CA Rotterdam, the Netherlands.

e-mail: m.vanroyen@erasmusmc.nl

M.E.v.R and E.I.V. contributed equally to this work. thick tissue slides, resulting in a two-dimensional (2D) tissue cross-section. In fact, little is known about the actual three-dimensional (3D) architecture of diseases such as PCa.

Three-dimensional microscopic tissue reconstruction has mostly been carried out by serial sectioning, staining and imaging of up to hundreds of $2 \mathrm{D}$ sections. $^{2-4}$ Although developments in image stacking have improved 3D reconstruction, serial slicing is laborious and sensitive to tissue deformations. ${ }^{5-8}$ Confocal laser scanning microscopy (CLSM) allows fluorescent 3D imaging without the need for sectioning, but is limited to depths of tens of micrometres, owing to scattering and absorption of emitted fluorescent light. Several protocols have recently been 
developed to optically clear tissue and thereby enhance the detection of emitted fluorescent light at increased tissue depths. ${ }^{9-14}$ Three-dimensional imaging by a combination of tissue clearance and CLSM has predominantly been used for investigation of mouse models, sometimes primarily labelled with green fluorescent protein. ${ }^{13,15,16}$ The aim of the current study was to investigate the feasibility of using tissue-clearing protocols and imaging techniques on fresh and formalin-fixed paraffin-embedded (FFPE) prostate specimens, in order to analyse both clinical and biological PCa features in their actual 3D context.

\section{Materials and methods}

CLINIC A L S PECIMENS

Prostate samples were derived from radical prostatectomies, performed at Erasmus Medical Centre, Rotterdam, the Netherlands between September 2014 and March 2016. The use of fresh and archival tissue samples for research purposes was approved by the local Medical Ethical Committee (MEC 2011-295 and 2011-296). After arrival at the pathology department, fresh samples of $5 \times 5 \mathrm{~mm}$ from both normal and tumour tissue were collected for 3D imaging $(N=13)$. From each sample, a 1 -mm slice was cut and processed for reference $5-\mu \mathrm{m}$ slice haematoxylin and eosin (HE) staining. Tissue samples for 3D imaging were sliced within $24 \mathrm{~h}$ in icecold phosphate-buffered saline (PBS) with a vibrating blade microtome Leica VT 1200 S (Leica, Rijswijk, the Netherlands). Slices with a thickness of 500$1000 \mu \mathrm{m}$ were fixed overnight in $1 \%$ formaldehyde in $\mathrm{PBS}$ at $4^{\circ} \mathrm{C}$. After fixation, tissue slices were washed in PBS, gradually incubated in methanol/ PBS up to $100 \%$ methanol, and stored at $-20^{\circ} \mathrm{C}$ until further processing.

STAINING OF FORMALIN-FIXED NON-EMBEDDED T I S S U E

Fixation, staining and clearing of fresh, nonembedded tissue was performed as described previously. ${ }^{17}$ Briefly, fixed tissue was rehydrated in methanol/PBS gradients up to $100 \% \mathrm{PBS}$, and blocked in PBS/1\% non-fat dry milk/0.4\% Triton X100. This was followed by incubation with RedDot2 (1:400; Biotium, Hayward, CA, USA) fluorescent nuclear dye, or primary antibodies targeting cytokeratin (CK) 5 (1:100, ab52635; AbCam, Cambridge, UK) and CK8-18 (1:100, MS-743S; Immunologic,
Duiven, the Netherlands) in PBS/1\% non-fat dry milk $/ 0.4 \%$ Triton $\mathrm{X}-100$ for a minimum of 7 days. After being washed in PBS/0.4\% Triton X-100, specimens were incubated with secondary Alexa 514-conjugated or Alexa 647-conjugated antibodies (1:200; Life Technologies, Bleiswijk, the Netherlands) in PBS/ $1 \%$ non-fat dry milk/0.4\% Triton X-100 for 7 days. Subsequent washing steps in PBS/0.4\% Triton X-100 were followed by gradient tissue dehydration in methanol. Prior to clearing, tissues were incubated with a mixture of 50\% methanol and 50\% 1:2 benzyl alcohol/benzyl benzoate (BABB) solution (Sigma, Zwijndrecht, the Netherlands) for $10 \mathrm{~min}$. Optical transparency was achieved after incubation with 100\% $\mathrm{BABB}$ solution for $10 \mathrm{~min}$. Tissue was stored in BABB at $4^{\circ} \mathrm{C}$ until imaging, for a maximum of 4 weeks.

STAINING OF FFPE TISSUE

For 3D imaging of archival FFPE prostate samples $(N=38)$, areas of interest were indicated on coverslips of HE-stained slides derived from diagnostic biopsies and radical prostatectomy specimens. Punches of thickness $500 \mu \mathrm{m}$ were collected from the corresponding FFPE block with tissue microarray punching needles (Estigen Tissue Science, Tartu, Estonia). Three-dimensional immunofluorescent (IF) tissue staining was performed according to the iDISCO protocol. ${ }^{12}$ Briefly, tissues were dewaxed overnight, and subsequently incubated in methanol for $60 \mathrm{~min}$, in 20\% dimethylsulphoxide (DMSO) and $20 \% \mathrm{H}_{2} \mathrm{O}_{2}$ in methanol at $4^{\circ} \mathrm{C}$, and then overnight in $20 \%$ DMSO in methanol. Tissue was rehydrated gradually in methanol/PBS, and this was followed by a blocking step with $0.2 \%$ Triton X-100/10\% DMSO/0.3 м glycine/PBS. Primary antibodies targeting CK5 (1:100) and CK8-18 (1:100) were incubated for 7 days in $0.2 \% /$ Tween $/ 20-10 \mu \mathrm{g} / \mathrm{ml}$ heparin $/ 5 \% \mathrm{DMSO} / 1 \%$ milk/PBS at $37^{\circ} \mathrm{C}$, and this was followed by washing in $0.2 \%$ Tween-20/20$10 \mu \mathrm{g} / \mathrm{ml}$ heparin/PBS, and incubation with secondary fluorescent Alexa 514-labelled or Alexa 647-labelled antibodies (1:200; Life Technologies) in $0.2 \%$ Tween-20/10 $\mu \mathrm{g} / \mathrm{ml}$ heparin $/ 5 \%$ DMSO $/ 1 \%$ milk/PBS for 7 days at $37^{\circ} \mathrm{C}$. Prior to clearing, tissue was washed in $0.2 \%$ Tween-20/10 $\mu \mathrm{g} / \mathrm{ml}$ heparin/ PBS overnight at $37^{\circ} \mathrm{C}$, dehydrated in methanol gradients at room temperature, and subsequently incubated in a mixture of 50\% methanol and 50\% BABB. Optical transparency was achieved in $100 \%$ BABB solution after $10 \mathrm{~min}$ of incubation. After clearing, tissue was stored for a maximum of 4 weeks in $\mathrm{BABB}$ at $4^{\circ} \mathrm{C}$ until imaging. 


\section{CONFOCAL MICROSCOPY}

For imaging, the samples were mounted in 100\% $\mathrm{BABB}$ in glass-bottomed microwell dishes (MatTek, Ashland, MA, USA), and covered with \#1 coverglasses (Menzel-Gläser, Braunschweig, Germany) to avoid direct contact of the microscope objective with BABB. Imaging was performed with an upright Leica SP5 confocal microscope (Leica Microsystems, Eindhoven, the Netherlands) equipped with $\mathrm{a} \times 20$ numerical aperture 1.0 APO water-dipping objective with a 1.95-mm working distance through a $170-\mu \mathrm{m}$ thick coverslip. Z-stacks were recorded with a $0.72-\mu \mathrm{m}$ pixel size and a 1-3- $\mu \mathrm{m}$ step-size in Z, by use of a 488$\mathrm{nm}$ argon laser and a 633-nm HeNe laser. Emission was detected in the 525-600-nm and 643-700-nm emission ranges, respectively. To compensate for loss of signal and optimize the collection of structural information, laser intensity and detector sensitivity were (semi-)automatically adjusted within a preset range. The settings were identical in each experiment. Images were deconvoluted with HUYGENS PROFESSIONAL software (SVI, Hilversum, the Netherlands). Threedimensional rendering and image editing were performed with FIJI (IMAGEJ 1.49s) and AMIRA (version 5.5.0; FEI, Hillsboro, OR, USA) software. ${ }^{18}$

\section{M M U N O H I S T O C HEM IS T R Y}

For subsequent 2D immunohistochemistry, cleared and three-dimensionally imaged tissues were returned to $100 \%$ methanol. Methanol was replaced by ethanol, and tissue was gradually rehydrated in PBS for re-embedding in paraffin. Five-micrometre FFPE sections of post-cleared tissues were deparaffinized and rehydrated with xylene and ethanol. Endogenous peroxidase was blocked in $0.3 \% \mathrm{H}_{2} \mathrm{O}_{2}$ in PBS, and heat-induced antigen retrieval was performed for $15 \mathrm{~min}$ in Tris-EDTA buffer ( $\mathrm{pH}$ 9; Klinipath, Duiven, the Netherlands). Primary antibodies targeting CK8-18 (1:500), CD31 (1:500; AbCam, Cambridge, MA, USA), Ki67 (1:200; Dako, Heverlee, Belgium) and vimentin (1:500; Dako) diluted in normal antibody diluent (APG-500; ScyTek Laboratories, Inc, West Logan, UT, USA) were incubated overnight at $4^{\circ} \mathrm{C}$, and visualized with the Envision kit (Dako). Slides were counterstained with haematoxylin, and visualized with an Olympus BX41 light microscope (Olympus, Zoeterwoude, the Netherlands).

\section{MOLECULAR ANALYSIS}

BABB-cleared tissues were placed in methanol and rehydrated in methanol/PBS gradients. DNA from tissue punches was isolated in lysis buffer (Promega, Leiden, the Netherlands) containing 5\% chelex (BioRad, Veenendaal, the Netherlands) with protease K (Promega) overnight at $56^{\circ} \mathrm{C}$. Protease deactivation was achieved by heating to $95^{\circ} \mathrm{C}$ for $10 \mathrm{~min}$. Polymerase chain reactions (PCRs) were performed with primers for the housekeeping genes TBXAS1, RAG1, PLZF and AF4 for 35 cycles at $60^{\circ} \mathrm{C}$. DNA from noncleared FFPE prostate samples served as a control. The DNA concentration and purity of six fresh cleared and six FFPE cleared tissues were measured with the Nanodrop system (Thermo Scientific, Wilmington, DE, USA). As a control, 0.5-mm punches of two non-cleared samples derived from the same patients were included.

\section{Results}

THREE - D I M EN S I O A L I M A G ING OF FRESH PROSTATE TISSUE

To evaluate the efficacy of tissue clearing, we first compared fluorescent staining in cleared versus noncleared prostate tissue samples $(N=4)$. Thick tissue slices were stained with RedDot 2 and imaged with CLSM. Nuclear RedDot2 signals could be detected throughout the whole BABB-cleared tissue slice of $800 \mu \mathrm{m}$. Without tissue clearing, the Reddot 2 signal was lost at $70 \mu \mathrm{m}$ within the tissue, and was only visible at the edges of the tissue up to a depth of $200 \mu \mathrm{m}$ in the image stack (Figure 1; Figure S1).

To gain insights into 3D normal and malignant prostate glandular architecture, we performed IF double-staining for CK5 and CK8-18. ${ }^{19,20}$ In general, CK5 labels pre-existing basal cells surrounding CK818-positive luminal epithelial cells in benign prostate glands. By using IF double-labelling, we were able to routinely image approximately $500-\mu$ m-thick tissues with sufficient signal throughout the stack. In the transition zone, 2D well-delineated round glandular structures were represented in three dimensions by organized acini (Figure 2A-D; Video S1). In the peripheral zone, 2D longitudinal tubule sections corresponded to 3D slit-like angulated tubular spaces (Figure 2E-H; Video S2).

THREE-DIMENSIONAL IMAGING OF FFPE PROSTATE TISSUE

The feasibility of clearing and 3D imaging on archival FFPE tissue samples was investigated on selected Gleason grade patterns of PCa as identified in 2D HE slides. PCa almost exclusively consists of CK8-18- 

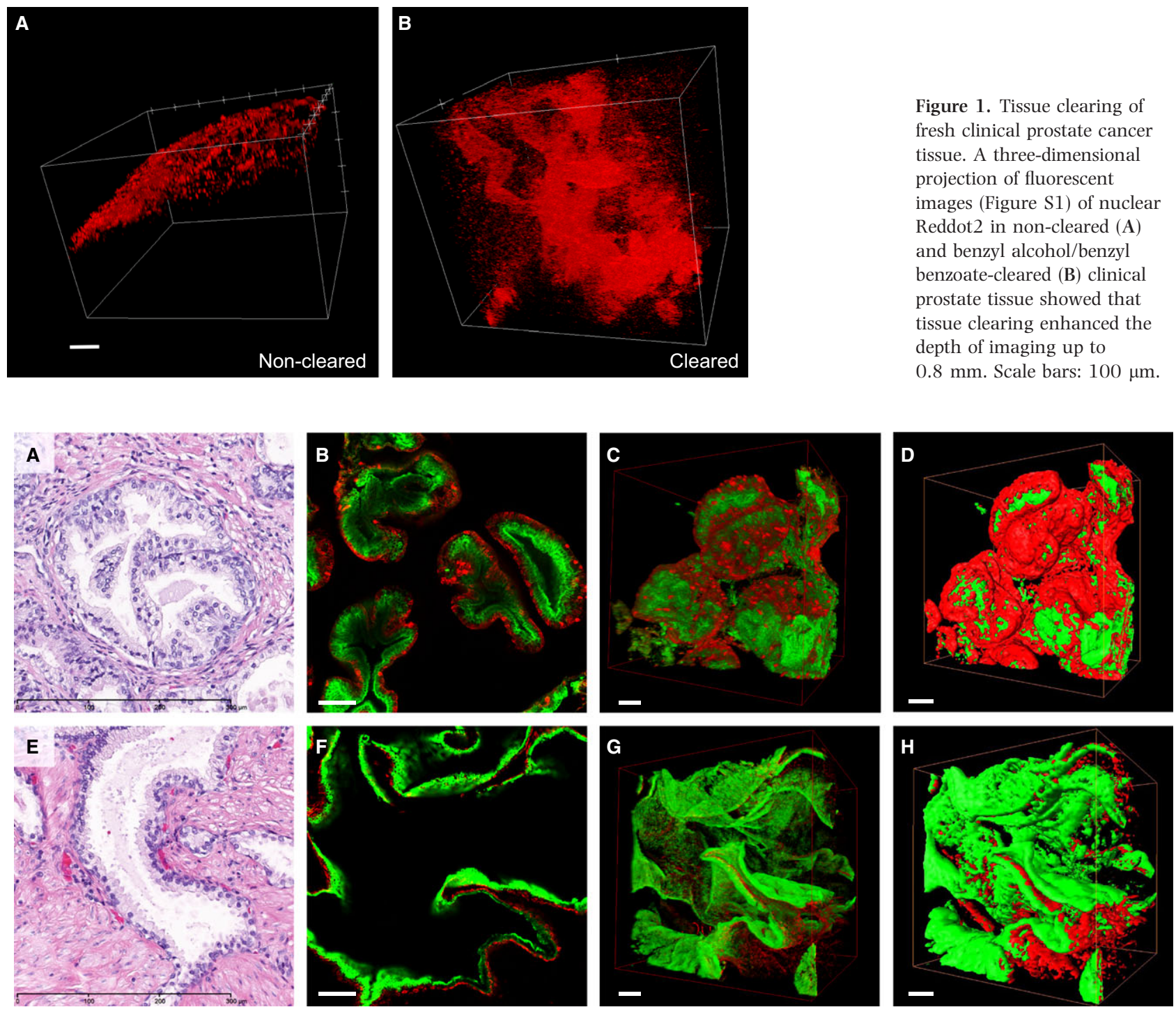

Figure 2. Three-dimensional (3D) imaging of antibody-stained fresh samples of benign prostate transition zone (A-D) and peripheral zone (E-H). A,E, Reference haematoxylin and eosin slide. B,F, Single two-dimensional optical plane of immunofluorescent cytokeratin (CK) 8-18labelled (green) and CK5-labelled (red), benzyl alcohol/benzyl benzoate-cleared tissue. C,G,D,H, Three-dimensional projection (C,G) and 3D binary projection (D,H) of approximately 500- $\mu \mathrm{m}$-thick tissue samples. Scale bars: $100 \mu \mathrm{m}$.

positive luminal cells, lacking a CK5-positive basal cell layer. ${ }^{19,20}$ Figure 3A-D demonstrates closely packed well-delineated glands corresponding to Gleason score $3+3=6$ PCa; on HE staining, branching and anastomosing of glands was not observed. However, 3D IF imaging showed that respective PCa glands represented an interconnecting glandular network (Video S3). A Gleason score 7 PCa consisting of well-delineated Gleason pattern 3 glands and Gleason pattern 4 with intraluminal glomeruloid epithelial proliferations is shown in Figure 3E-H (Video S4). Three-dimensional projection revealed that the glandular structures with intraluminal glomeruloid proliferations were continuous with well-delineated Gleason grade 3 glands, which was not appreciated in the corresponding HE slide. Figure 3J-L depicts an area of ill-defined Gleason pattern $4 \mathrm{PCa}$ glands. Three-dimensional analysis revealed that these structures represented a highly anastomosing meshwork of small-sized tubules and cords of malignant cells (Video S5). The interobserver variability of ill-defined tumour glands is relatively poor, as discriminating them from tangentially sectioned Gleason grade 3 or Gleason grade 5 glands is often difficult. ${ }^{21}$ Threedimensional imaging was able to separate welldelineated Gleason grade 3 glands (Figure 3A-D) 

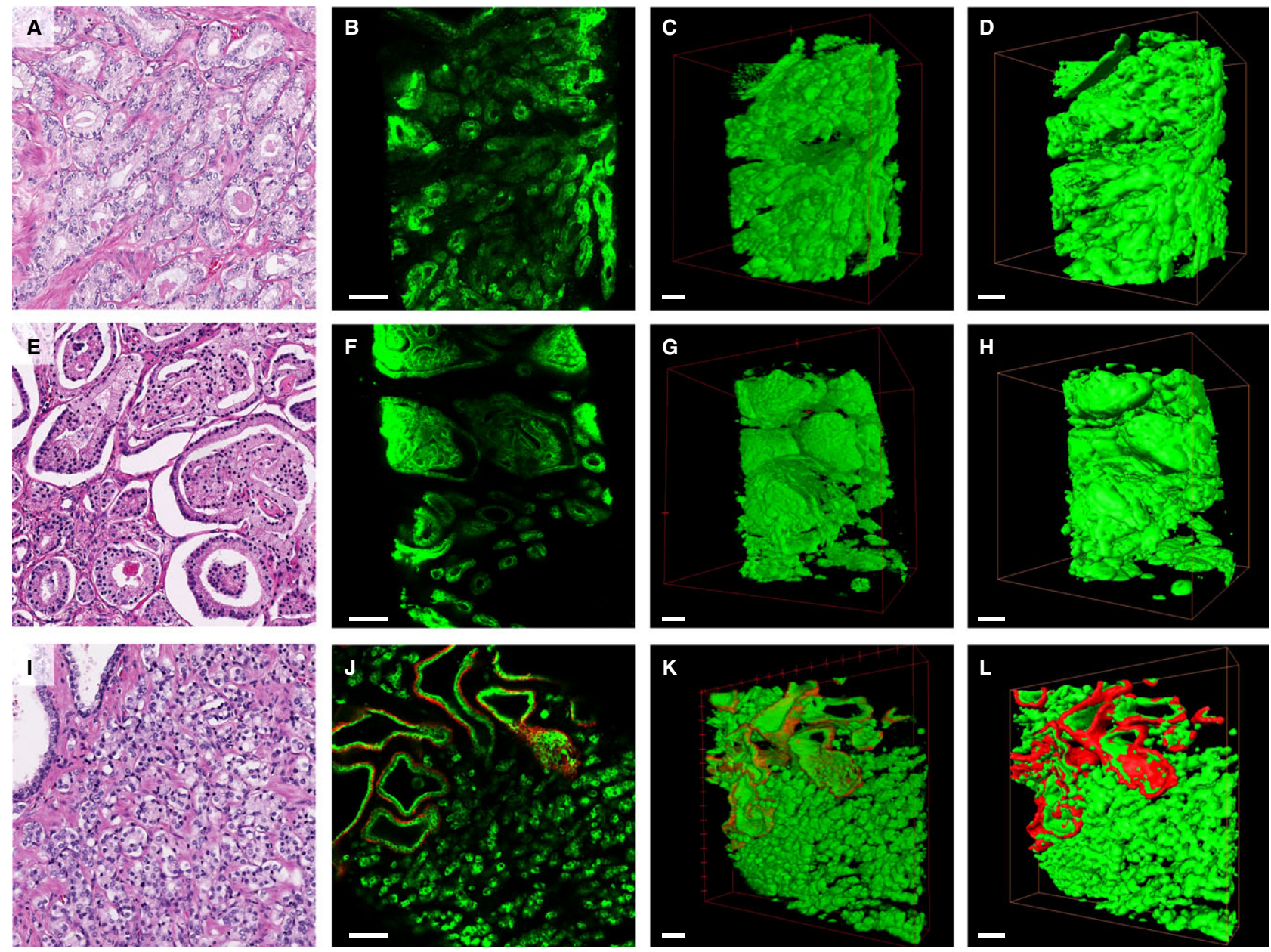

Figure 3. Three-dimensional (3D) pathology of prostate cancer growth patterns. A-D, Well-delineated Gleason grade 3 prostate cancer with regular 3D glandular interconnections. E-H, Well-delineated Gleason grade 3 and glomeruloid Gleason grade 4 glands. Three-dimensional spatial transitions between both structures exist. I-L, Three-dimensional projection of an ill-defined Gleason grade 4 gland demonstrates a highly interconnecting meshwork of small-sized tubules and cords. Vertical panels from left to right represent a reference haematoxylin and eosin slide, a single 2D optical plane of immunofluorescent cytokeratin (CK) 8-18-labelled (green) and CK5-labelled (red) benzyl alcohol/benzyl benzoate-cleared tissue, and original 3D and 3D binary projections. Scale bars: $100 \mu \mathrm{m}$.

from an ill-defined Gleason grade 4 growth pattern (Figure 3J-L) on the basis of the large number of small anastomosing tubules in the latter.

TISSUE PROCESSING AFTER 3 D IMAGING

To determine whether 2D immunohistochemistry and molecular analysis were still possible after 3D IF and BABB clearing, we rehydrated and paraffin-embedded three-dimensionally imaged tissue samples. Tissue morphology on HE staining before and after 3D processing was comparable. Conventional immunohistochemical staining for CK8-18, CD31, Ki67 and vimentin demonstrated that specific protein labelling after 3D imaging was still feasible (Figure 4A).
However, respective stainings demonstrated cytoplasmic labelling of basal epithelial cells in fresh tissue, which was attributable to shared primary antibody species with the CK5 antibody that was used for 3D IF imaging prior to conventional 2D staining (Figure S2C). After BABB clearing and 3D imaging, the DNA yield was sufficient for multiple molecular analysis (range 68.4-202.7 ng/ $\mu \mathrm{l}$ ), with minor loss of purity (range $0.7-1.1 \mathrm{ng} / \mu \mathrm{l}$ ) (Table 1). DNA quality was sufficient for PCR amplification and analysis, with some degradation as compared with non-cleared control tissue (Figure 4B; Figure S2D). In contrast, RNA isolation resulted in small quantities of mostly degraded RNA (data not shown), which was not sufficient for RNA analysis. 

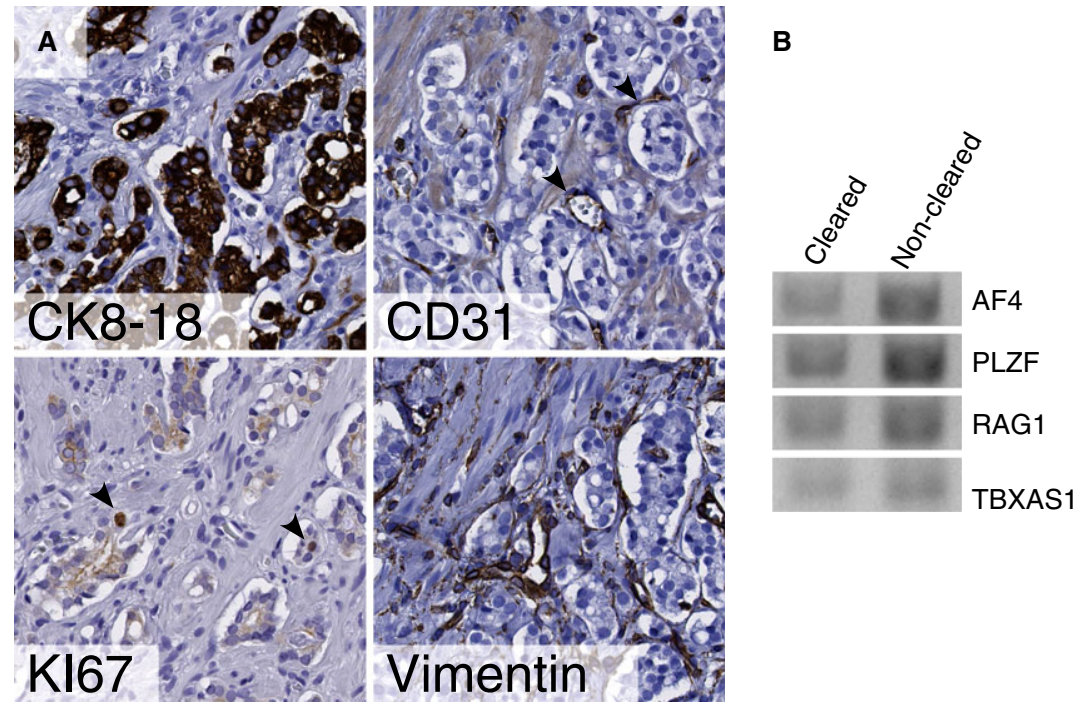

Figure 4. Processing of cleared formalin-fixed paraffin-embedded archival prostate cancer tissue. A, Immunohistochemical cytokeratin (CK) 8-18, CD31, Ki67 and vimentin staining of cleared, re-embedded tissue sections demonstrates labelling of luminal epithelium, endothelium, proliferating cells, and stromal cells (arrowheads). B, Routine quality polymerase chain reaction on DNA isolated from cleared tissue samples shows the applicability of molecular analysis after three-dimensional imaging, despite some degradation as compared with control noncleared tissue.

Table 1. DNA yield and purity of cleared and uncleared tissues

\begin{tabular}{lll}
\hline & Yield in $n g / \mu l(S D)$ & Purity (SD) \\
\hline FFPE cleared $(n=6)$ & $68.4(13.9)$ & $0.7(0.0)$ \\
\hline FFPE uncleared $(n=2)$ & $145.8(41.2)$ & $1.0(0.1)$ \\
\hline Fresh cleared $(n=6)$ & $202.7(96.2)$ & $1.1(0.2)$ \\
\hline Fresh uncleared $(n=2)$ & $163.7(2.9)$ & $1.1(0.0)$ \\
\hline
\end{tabular}

FFPE, formalin-fixed paraffin-embedded; SD, standard deviation.

\section{Discussion}

In this study, we have demonstrated that BABBmediated tissue clearing can successfully be applied for 3D imaging of fresh and FFPE clinical prostate specimens up to a depth of $\sim 800 \mu \mathrm{m}$. The feasibility of this methodology for FFPE tissues, in particular, offers the opportunity to study specific pathological and biological features of interest in their 3D context. Moreover, 3D imaging has been raised as a novel diagnostic tissueprocessing methodology. ${ }^{22}$ We have also shown that, after 3D imaging, tissue samples are still suitable for routine 2D processing, including immunohistochemistry and molecular analysis, which is relevant in cases of unique tissue samples and/or small tissue fragments.

We were able to image small nuclear dyes as well as antibodies, visualizing the $3 \mathrm{D}$ microscopic structure of both benign and malignant human prostate samples. We set out to develop 3D imaging in clinical prostate tissues to obtaing more profound insights into the $\mathrm{PCa}$ growth patterns underlying the current Gleason grading system. Although the current study was not aimed at performing detailed characterization of various growth patterns, we were able to preliminarily interpret a subset of growth patterns. That is, we have shown that: (i) Gleason grade 3 glands are branching and connecting; (ii) glomeruloid structures are continuous with delineated Gleason grade 3 glands; and (iii) ill-defined Gleason grade 4 glands represent small tubules with more frequent interconnections.

Several approaches have been applied for 3D imaging of tissues. Although these technologies have proven to be valuable for non-invasive imaging of tissues, they are not based on labelling and visualization of specific (disease-related) structures [e.g. magnetic resonance imaging (MRI), (micro-)Raman spectroscopy, and optical coherence tomography], or do not reveal sufficient detail of microscopic structures in tissues (MRI and fluorescence tomography). Tissue-clearing techniques enable the visualization of (disease)-specific structures while still maintaining the microscopic detail, as we have demonstrated in this study, thus offering an opportunity for diagnostic pathology in the future. Importantly, Torres et al. demonstrated comparable morphology between 
reference HE 2D slides and BABB-cleared 800- $\mu$ mthick samples imaged by multiphoton microscopy with pseudocolouring. ${ }^{22}$ The advantages of such an approach would be that tissue samples remain intact and that no material is lost during tissue processing. We have shown that 2D immunohistochemistry and molecular analysis can still be performed on optically cleared tissue used for 3D imaging. With the current technological advancements in view, including deep tissue scanning and fast staining protocols, imaging of intact tissue samples might become an alternative to traditional 2D HE-stained tissue slides and corresponding tissue loss in the future.

Although passive antibody diffusion in complete tissue samples is time-consuming and is a limitation in this study, significant improvements in the staining protocol, including staining in high-pressure conditions, could well result in improved antibody penetration, and enable the visualization of 3D structures in even larger tissue samples. Second, the interpretation and quantification of both $3 \mathrm{D}$ and 2D growth patterns is subjective, emphasizing the need for more objective image analysis approaches to more objectively describe and separate tissue structures.

In conclusion, we have described a methodology for clearing and 3D imaging of fresh and FFPE prostate specimens up to a depth of $800 \mu \mathrm{m}$. This methodology allows 3D analysis of an unlimited range of normal and pathological structures in clinical tissue specimens. The procedure is compatible with subsequent tissue sectioning, 2D (immuno)histochemistry, and molecular analysis, allowing multiple analyses on limited tissue samples.

\section{Acknowledgements}

We thank Peggy Atmodimedjo and Carolina Avença Valente for performing the DNA isolation and PCR.

\section{Author contributions}

M. E. van Royen, E. I. Verhoef and G. J. L. H. van Leenders designed the study, performed the research, and wrote the paper. C. F. Kweldam, W. A. van Cappellen, G.-J. Kremers and A. B. Houtsmuller contributed essential tools. M. E. van Royen, E. I. Verhoef and W. A. van Cappellen analysed the data.

\section{Conflicts of interest}

The authors declare no conflicts of interest.

\section{References}

1. Epstein JI, Egevad L, Amin MB et al. The 2014 International Society of Urological Pathology (ISUP) Consensus Conference on gleason grading of prostatic carcinoma: definition of grading patterns and proposal for a new grading system. Am. J. Surg. Pathol. 2015; 40; 244-252.

2. Marchio C, Sapino A, Arisio R, Bussolati G. A new vision of tubular and tubulo-lobular carcinomas of the breast, as revealed by 3-D modelling. Histopathology 2006; 48; 556-562.

3. Onozato ML, Klepeis VE, Yagi Y, Mino-Kenudson M. A role of three-dimensional (3D)-reconstruction in the classification of lung adenocarcinoma. Anal. Cell Pathol. (Amst.) 2012; 35; 79-84.

4. Booth ME, Treanor D, Roberts N, Magee DR, Speirs V, Hanby AM. Three-dimensional reconstruction of ductal carcinoma in situ with virtual slides. Histopathology 2015; 66; 966-973.

5. Sun L, Wang D, Zubovits JT, Yaffe MJ, Clarke GM. An improved processing method for breast whole-mount serial sections for three-dimensional histopathology imaging. Am. J. Clin. Pathol. 2009; 131; 383-392.

6. Norton KA, Namazi S, Barnard N et al. Automated reconstruction algorithm for identification of 3D architectures of cribriform ductal carcinoma in situ. PLoS ONE 2012; 7; e44011.

7. Gibson E, Gaed M, Gomez JA et al. 3D prostate histology reconstruction: an evaluation of image-based and fiducial-based algorithms. Med. Phys. 2013; 40; 093501-1-093501-15.

8. Gibson E, Gaed M, Gomez JA et al. 3D prostate histology image reconstruction: quantifying the impact of tissue deformation and histology section location. J. Pathol. Inform. 2013; 4; 31.

9. Hama H, Kurokawa H, Kawano $\mathrm{H}$ et al. Scale: a chemical approach for fluorescence imaging and reconstruction of transparent mouse brain. Nat. Neurosci. 2011; 14; 1481-1488.

10. Erturk A, Becker K, Jahrling N et al. Three-dimensional imaging of solvent-cleared organs using 3DISCO. Nat. Protoc. 2012; 7; 1983-1995.

11. Chung K, Wallace J, Kim SY et al. Structural and molecular interrogation of intact biological systems. Nature 2013; 497; 332-337.

12. Renier N, Wu Z, Simon DJ, Yang J, Ariel P, Tessier-Lavigne M. iDISCO: a simple, rapid method to immunolabel large tissue samples for volume imaging. Cell 2014; 159; 896-910.

13. Tainaka K, Kubota SI, Suyama TQ et al. Whole-body imaging with single-cell resolution by tissue decolorization. Cell 2014; 159; 911-924.

14. Richardson DS, Lichtman JW. Clarifying tissue clearing. Cell 2015; 162()$; 7$.

15. Scott GD, Blum ED, Fryer AD, Jacoby DB. Tissue optical clearing, three-dimensional imaging, and computer morphometry in whole mouse lungs and human airways. Am. J. Respir. Cell Mol. Biol. 2014; 51; 43-55.

16. Yang B, Treweek JB, Kulkarni RP et al. Single-cell phenotyping within transparent intact tissue through whole-body clearing. Cell 2014; 158; 945-958.

17. Yokomizo T, Yamada-Inagawa T, Yzaguirre AD, Chen MJ, Speck NA, Dzierzak E. Whole-mount three-dimensional imaging of internally localized immunostained cells within mouse embryos. Nat. Protoc. 2012; 7; 421-431.

18. Schindelin J, Arganda-Carreras I, Frise E et al. Fiji: an opensource platform for biological-image analysis. Nat. Methods 2012; 9; 676-682.

19. Okada H, Tsubura A, Okamura A et al. Keratin profiles in nor$\mathrm{mal} /$ hyperplastic prostates and prostate carcinoma. Virchows Arch. A Pathol. Anat. Histopathol. 1992; 421; 157-161. 
20. van Leenders G, Dijkman H, Hulsbergen-van de Kaa C, Ruiter D, Schalken J. Demonstration of intermediate cells during human prostate epithelial differentiation in situ and in vitro using triple-staining confocal scanning microscopy. Lab. Invest. 2000; 80; 1251-1258.

21. Zhou M, Li J, Cheng L et al. Diagnosis of 'poorly formed glands' Gleason pattern 4 prostatic adenocarcinoma on needle biopsy: an interobserver reproducibility study among urologic pathologists with recommendations. Am. J. Surg. Pathol. 2015; 39; 1331-1339.

22. Torres R, Vesuna S, Levene MJ. High-resolution, 2- and 3dimensional imaging of uncut, unembedded tissue biopsy samples. Arch. Pathol. Lab. Med. 2014; 138; 395-402.

\section{Supporting Information}

Additional Supporting Information may be found in the online version of this article:
Figure S1. Tissue clearing of fresh clinical prostate cancer tissue.

Figure S2. Processing of cleared fresh fixed tissue.

Video S1. 3D binary projection of cleared prostate transition zone; CK8-18 (green) and CK5 (red).

Video S2. 3D binary projection of cleared prostate peripheral zone; CK8-18 (green) and CK5 (red).

Video S3. 3D binary projection of cleared Gleason grade 3; CK8-18 (green) and CK5 (red).

Video S4. 3D binary projection of cleared Gleason grade 3 and glomeruloid Gleason grade 4; CK8-18 (green) and CK5 (red).

Video S5. 3D binary projection of cleared ill-formed Gleason grade 4; CK8-18 (green) and CK5 (red). 